\title{
Sanidad y redes sociales: ¿qué entidades son más activas y con cuáles interactúa más el público?
}

\author{
Healthcare and social networks: Which organizations \\ are more active and with which does the public \\ interact more?
}

\author{
Lorena Busto-Salinas
}

Cómo citar este artículo:

Busto-Salinas, Lorena (2019). "Sanidad y redes sociales: ¿qué entidades son más activas y con cuáles interactúa más el público?". El profesional de la información, v. 28, n. 2, e280215.

https://doi.org/10.3145/epi.2019.mar.15

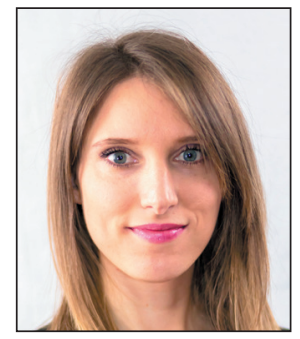

\section{Lorena Busto-Salinas}

Artículo recibido el 17-12-2018

http://orcid.org/0000-0003-0768-8349

Universidad de Burgos

Facultad de Ciencias Económicas y Empresariales

Plaza de la Infanta Dña. Elena, s/n. 09001 Burgos, España

Ibusto@ubu.es

\section{Resumen}

Los artículos que analizan la presencia y la actividad de los hospitales en redes sociales son abundantes, pero los que estudian otras organizaciones vinculadas con la salud son muy escasos. En esta investigación se han examinado los perfiles en Facebook, Twitter, YouTube e Instagram de 240 entidades relacionadas con la sanidad. Se ha comprobado que las aseguradoras de salud están más presentes en las redes sociales que el resto, seguidas por los fabricantes de productos sanitarios y las empresas de tecnología. A la cola se encuentran los laboratorios farmacéuticos, las empresas de productos biomédicos y, sobre todo, los hospitales. No obstante, se ha hallado que los usuarios interactúan más con los hospitales, a pesar de que son menos asiduos y menos activos en estas plataformas. Se confirma así que una mayor presencia y actividad en las redes sociales no se correlaciona con una mayor interacción por parte del público.

\section{Palabras clave}

Comunicación para la salud; Información de salud; Sanidad; E-salud; Relaciones públicas; Comunicación corporativa; Redes sociales; Medios sociales; Facebook; Twitter; YouTube; Instagram.

\begin{abstract}
Previous research on the presence and activity of hospitals in social media is abundant, but that is not the case of other health-related organizations. In this research we have examined the profiles on Facebook, Twitter, YouTube and Instagram of 240 health-related entities. It has been proven that health insurers are more present in social networks than the rest, followed by manufacturers of healthcare products, technology companies, pharmaceutical laboratories, biomedical products companies and hospitals. Nevertheless, users interact more with hospitals, although they are less frequent and less active on these platforms. These findings confirm that a greater presence and activity in social media does not correlate with a greater interaction by the public.
\end{abstract}

\section{Keywords}

Health communication; Health information; Healthcare; E-health; Public relations; Corporate communication; Social networks; Social media; Facebook; Twitter; YouTube; Instagram. 


\section{Introducción y estado de la cuestión}

Internet se ha convertido en una de las principales fuentes de información sobre salud y sanidad. Una muestra de ello es que el $72 \%$ de los internautas adultos de Estados Unidos busca información relacionada sobre salud en la Red (Fox, 2014). El uso de las redes sociales con esa intención ha aumentado en consonancia y Facebook se ha convertido en el segundo lugar más recurrente en la búsqueda de información sobre salud en internet (el 59\% de los pacientes lo utilizan), por detrás de las webs especializadas (usadas por el 70\% de los pacientes) (Health Union, 2016). Ahora bien, a la hora de compartir información sobre salud, Facebook se sitúa en primer lugar: el $87 \%$ de los pacientes lo usa para este fin y el $91 \%$ asegura que las comunidades online influyen en sus decisiones relacionadas con la salud (WEGO Health, 2017).

Los pacientes no sólo quieren interactuar con otros internautas, sino que desean que los médicos e instituciones sanitarias participen en la creación de webs o de perfiles en redes sociales a las cuales puedan acudir para informarse (Health Research Institute, 2012). Mantener perfiles apropiados en medios sociales puede ser un buen indicador de la calidad de un hospital y de la satisfacción de los usuarios. Analizando 40 hospitales del entorno de Nueva York, Timian et al. (2013) encontraron que el número de "me gusta" en Facebook tiene una asociación negativa con la tasa de mortalidad en 30 días y una asociación positiva con las recomendaciones de los clientes. Un mayor uso de Facebook y una mejor puntuación en esta red social también se relaciona con una media de readmisiones más baja (Glover et al., 2015).

\subsection{Presencia en redes sociales de entidades relacionadas con la salud}

La bibliografía sobre la presencia y actividad en redes sociales de las organizaciones relacionadas con la salud es muy dispar dependiendo del tipo de entidad. La mayoría de las investigaciones sobre el ámbito farmacéutico se centran en personas individuales y sobre todo en estudiantes (o en su defecto, profesores universitarios) [Benetoli et al. (2017) hacen un exhaustivo repaso a los artículos publicados al respecto]. Alkhateeb, Clauson y Latif (2011) son de los pocos que ofrecen resultados con profesionales farmacéuticos. A través de una encuesta realizada en 2009 en una convención anual de una asociación de farmacia en West Virginia (Estados Unidos), detectaron un uso considerable de YouTube (74\%), Wikipedia (72\%), Facebook (50\%) y blogs (26\%), aunque básicamente con fines personales.

En Inglaterra, el 72,8\% de los farmacéuticos encuestados por Crilly et al. (2018) mantiene un perfil en alguna red social, y de ellos, el 77,5\% entra cada día. Facebook es la plataforma más recurrente, seguida de LinkedIn, YouTube, Instagram y Twitter. De los que usan las redes sociales, el 57,2\% lo hace con fines profesionales.

Tyrawski y DeAndrea (2015) analizaron la presencia de la publicidad directa de las farmacéuticas. Salvo en un caso, todas redireccionan a una red social al menos desde su web. Twitter es la más usada (en el 93\% de los casos), seguida de Facebook (66\%), YouTube (66\%) y LinkedIn (60\%). Observan que alrededor del 40\% de las publicaciones en Facebook, Twitter y YouTube responden a lo que se considera help-seeking advertisement (que implica crear conciencia sobre una condición o enfermedad médica y alentar a la audiencia a buscar remedios con su médico), y es más habitual en YouTube y en Twitter que en Facebook. En el 92\% de los posts, las empresas farmacéuticas son las autoras del contenido.

La bibliografía es más abundante en el caso de los hospitales, y desde aproximadamente 2014 las investigaciones muestran una tendencia al alza en el uso de redes sociales. Por ejemplo, en un estudio de Griffis et al. (2014), se observa que el $94,41 \%$ de los hospitales de Estados Unidos tienen una página en Facebook y el 50,82\% en Twitter. Prácticamente todos poseen un alojamiento en Yelp $(99,14 \%)$ y en Foursquare $(99,41 \%)$.

Glover et al. (2015) observan que el 88\% tiene una página en Facebook, ya sea propia o compartida con un grupo sanitario.

Smith (2017) consigue cifras muy similares: 95\% para Facebook y 75\% para Twitter y YouTube tomando como referencia la lista Truven Health Analytics' 100 Top hospitals de 2015; muy por detrás se queda Google+ (19\%). De media, concluye Smith, los hospitales están presentes en 3,3 plataformas.

En cuanto a los centros hospitalarios infantiles, todos ellos mantienen perfiles en Facebook y Twitter y la mayoría usan YouTube (82\%), Google+ (53\%) y Pinterest (69\%) (Wong et al., 2016). En Canadá el 58\% de los hospitales están presentes en Facebook (Medina-Aguerrebere; Buil-Gazol; Heath, 2015). De los 15 hospitales privados de Ankara (Turquía) analizados por Gülnur İlgün y Özgür Uğurluoğlu (2018), el 100\% usa Facebook, por delante de Twitter (93,3\%), Instagram $(66,7 \%)$, YouTube $(33,3 \%)$ y Linkedln $(26,7 \%)$.

En todos los continentes, la red social Facebook es la que mayor presencia tiene, seguida por Twitter y YouTube. Las menos utilizadas son Vimeo y Flickr, según se desprende de un estudio de los hospitales que ocupan las 100 primeras posiciones en cada continente en el Ranking web de hospitales del mundo de Webometrics (Benítez-Berrocal; Faba-Pérez, 2016).

En España el 34,2\% de los 787 hospitales del país tenían al menos un perfil en Facebook, Twitter o YouTube en diciembre de 2015, una cifra sensiblemente más baja si se compara con los resultados otorgados por los centros de Estados Unidos y Europa occidental (Martinez-Millana et al., 2017). A pesar de ello es diez veces mayor de lo que se contabilizaba en 2011 (Martinez-Millana et al., 2017). 
Aunque se reduzca la muestra a los hospitales más reputados de España, las cifras siguen siendo bastante más bajas que en otros países. De los 100 hospitales que se incluyen en el Monitor de reputación sanitaria de 2015, sólo el 27\% tiene un perfil propio de Facebook, el 26\% de Twitter y el $20 \%$ de YouTube. Uniendo los canales propios con los compartidos (por ejemplo, con consejerías de sanidad, otros centros del grupo hospitalario, etc.) el 50\% de los hospitales españoles que figuran en el Monitor están presentes en alguna de esas tres redes sociales (Costa-Sánchez; Túñez-López; Videla-Rodríguez, 2016).

Si se consulta el Ranking mundial de hospitales 2015, elaborado por el Consejo Superior de Investigaciones Científicas de España -CSIC-, y se analizan los 100 mejores de España, se observa que las redes sociales más utilizadas por los hospitales españoles son Twitter (68\%), Facebook (62\%) y YouTube (61\%); las que menos son Pinterest (4\%) y Flickr (3\%) (Medina-Aguerrebere, 2018).

Pese a todo, Costa-Sánchez y Míguez-González (2018), comparando el Hospital Sant Joan de Déu con la Clínica Universidad de Navarra (España), comprobaron que una mayor actividad en redes sociales no implica una mayor interacción con el público.

\section{Justificación y objetivos}

Existen numerosos estudios sobre la presencia de hospitales en redes sociales, pero no se ha analizado con la misma precisión la presencia de otras entidades relacionadas con la salud - como laboratorios farmacéuticos, aseguradoras de salud, empresas de dispositivos biomédicos, etc. - En consecuencia, no se puede hacer una radiografía de las fuentes de información sobre salud disponibles en redes sociales y su consumo por parte de los usuarios. Para paliar esta situación, este estudio pretende dar respuesta a las siguientes cuestiones:

- ¿Qué entidades relacionadas con la salud tienen una mayor presencia en redes sociales?

- ¿Qué entidades relacionadas con la salud son más activas en redes sociales?

- ¿Con qué entidades los usuarios interaccionan más en redes sociales?

- ¿Existe una correlación entre la presencia y la actividad en redes sociales y la interacción de la audiencia?

\section{Metodología}

Para dar respuesta a las preguntas planteadas, se ha utilizado el Monitor de reputación sanitaria (MRS) de 2017 (Análisis e Investigación, 2017). En este ranking aparecen:

- los 100 hospitales más reputados del país (74 públicos y 26 privados);

- 60 laboratorios farmacéuticos;

- 40 fabricantes de productos sanitarios;

- 25 aseguradoras de salud;

- 10 empresas de tecnología;

- 5 empresas de dispositivos biomédicos.

En total, se han estudiado 240 entidades relacionadas con la salud de 6 ámbitos diferentes.

Los hospitales se enmarcan dentro de la estructura de la atención especializada, que es el nivel que comprende las "actividades asistenciales, diagnósticas, terapéuticas y de rehabilitación y cuidados, así como aquéllas de promoción de la salud, educación sanitaria y prevención de la enfermedad" (España, 2003).

En la actualidad existen en España 799 hospitales en funcionamiento (Ministerio de Sanidad, 2018).

Según su dependencia funcional (Ministerio de Sanidad, 2018):

- el $41,7 \%$ son privados con ánimo de lucro;

- el 40,3\% públicos civiles;

- el 15,3\% privados sin ánimo de lucro;

- el 2,5\% forma parte de mutuas de accidentes de trabajo y enfermedades profesionales (MATEP);

- el 0,3\% son de Defensa.

Los laboratorios farmacéuticos pueden dividirse en (España, 2010):

- fabricantes: se dedican a la fabricación de medicamentos o medicamentos en investigación;

- importadores: se encargan de realizar los análisis cualitativos y cuantitativos para su importación;

- titulares de la autorización de comercialización: son responsables de la comercialización con instalaciones en España, propias o contratadas, para almacenar y distribuir medicamentos

Los fabricantes de productos sanitarios necesitan una licencia previa de funcionamiento, otorgada por la Agencia Española de Medicamentos y Productos Sanitarios si se trata de productos generales, o por las autoridades sanitarias de 
la comunidad autónoma correspondiente en el caso de productos a medida. Sus productos están regulados por el Real Decreto 1591/2009, de 16 de octubre, por el que se regulan los productos sanitarios (España, 2009). A partir del 26 de mayo de 2020, tendrán que cumplir con el Reglamento UE 2017/745 (Unión Europea, 2017). El Monitor de reputación sanitaria diferencia entre fabricantes de productos sanitarios y empresas de dispositivos biomédicos. En el primer grupo están por ejemplo B. Braun, 3M o Coloplast, y en el segundo Depuy Synthes, KLS Martin o Stryker.

Las primas de las aseguradoras de salud suponen el segundo gasto más importante de los españoles en el terreno de la salud (con el 16,6\% del total), sólo superado por los productos farmacéuticos (20,2\%) (Unespa, 2017). El 9\% de la población española tiene un seguro de salud individual, aunque asciende al $21,3 \%$ si se suman las mutualidades de funcionarios, los asegurados por otros colectivos (como empresas) y los seguros de reembolsos de gastos médicos (Unespa, 2017).

Las empresas de tecnología en el ámbito de la salud pretenden

"poner al alcance de todos tecnologías, productos y servicios que mejoran la prevención, el diagnóstico, el tratamiento y el control y seguimiento de las enfermedades" (Fenin, s.f.).

En España la Federación Española de Empresas de Tecnología Sanitaria (Fenin) agrupa a las empresas españolas de este sector, coordina y defiende sus intereses generales y ostenta su representación ante las autoridades autonómicas, nacionales y europeas (Fenin, s.f.).

Para hallar la presencia en redes sociales de estas organizaciones se han consultado las cuentas en Facebook, Twitter, YouTube e Instagram de todas las entidades que aparecen en el Monitor de reputación sanitaria. Junto con Whatsapp son las redes sociales más usadas en España (IAB Spain, 2017). Únicamente se han tenido en cuenta los perfiles propios de cada centro, no aquellos compartidos (por ejemplo, de la consejería de sanidad, del área de salud, del grupo sanitario, etc.), así como los que se encuentran actualizados, al menos en el último año, con la excepción por sus características de YouTube. La recogida de información ha tenido lugar durante los meses de agosto y septiembre de 2018. Los resultados se han introducido en el programa estadístico SPSS y se han realizado diversos análisis para conocer su relación. La significación en las pruebas estadísticas se ha fijado en igual o menor de 0,05. De esta forma se puede establecer que existe una relación estadística y que los resultados no son fruto del azar.

\section{Resultados}

\subsection{Presencia en redes sociales}

Sumando las 240 entidades analizadas se han detectado 471 perfiles. La red social más utilizada es Twitter: el 59,2\% $(\mathrm{N}=142)$ de las instituciones tiene una cuenta actualizada en esta plataforma. Muy cerca se halla YouTube, que aglutina al 56,7\% ( $N=136)$. En tercera posición se encuentra Facebook (51,3\%; $\mathrm{N}=123$ ) y en cuarta, Instagram (29,2\%; N=70). El 78\% de las aseguradoras de salud mantienen un perfil en al menos una red social. Les siguen los fabricantes de productos sanitarios con el $69 \%$, las empresas de tecnología con el $67 \%$, y los laboratorios farmacéuticos con el $65 \%$. Muy por detrás se hallan las empresas de dispositivos biomédicos (45\%) y los hospitales (23\%).

En el gráfico 2 se puede ver el porcentaje de presencia de las agrupaciones analizadas en cada red social. La tasa más alta la proporcionan las aseguradoras de salud (con una media de 3,12 cada una), seguida por los fabricantes de productos sanitarios $(2,75)$, las empresas de tecnología $(2,70)$ y los laboratorios farmacéuticos $(2,58)$. Con bastante diferencia se hallan las empresas de dispositivos biomédicos $(1,80)$ y, sobre todo, los hospitales $(0,92)$.

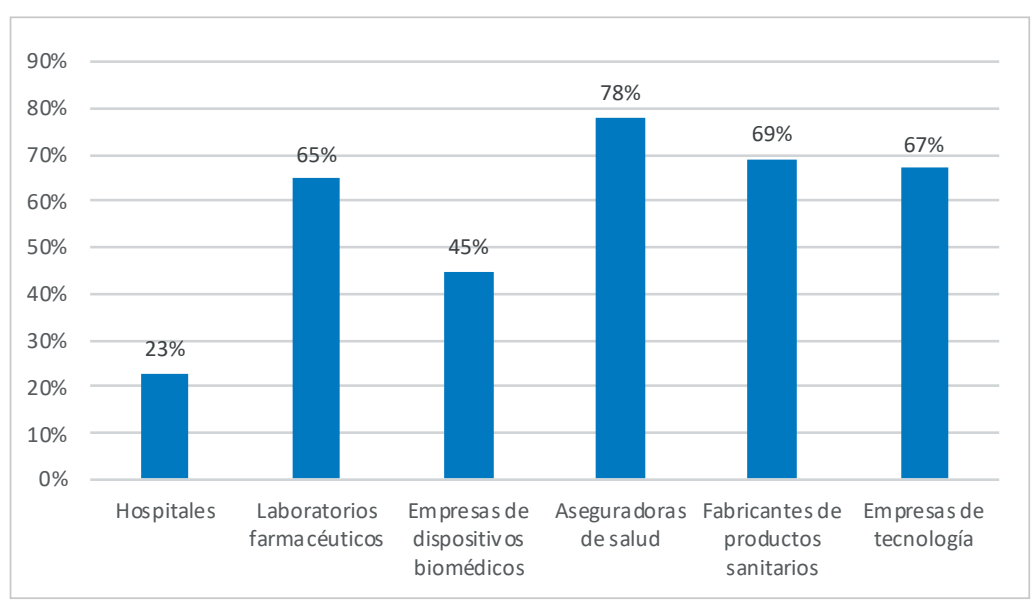

Gráfico 1. Porcentajes de presencia en al menos una red social.

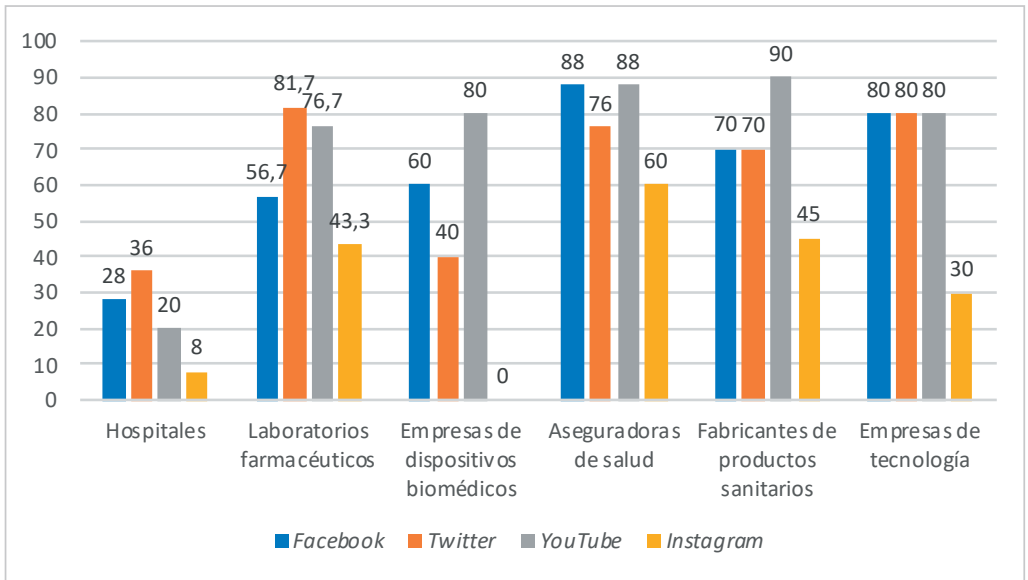

Gráfico 2. Porcentajes de presencia en cada red social. 


\subsection{Actividad en Facebook}

Las empresas de tecnología tienen estadísticamente más "me gusta" a sus perfiles en Facebook que cualquier otro tipo de corporaciones. De media arrojan 229.155, frente al promedio de 117.475 que obtienen los siguientes (los fabricantes de productos sanitarios) y los 6.545 de los últimos (los hospitales). Entre unos y otros se encuentran las empresas de dispositivos biomédicos (113.839), los laboratorios farmacéuticos (60.624) y las aseguradoras de salud (22.015). La media general es 65.299. La relación es significativa al 0,004.

Al igual que en el número de "me gusta" a la página, las empresas de tecnología también poseen más seguidores en sus perfiles. De media, cuentan con 228.182, frente al promedio general, que baja hasta los 65.258. En segundo lugar, se sitúan los fabricantes de productos sanitarios con 117.402, y las empresas de dispositivos biomédicos con 113.909. A la cola se hallan los laboratorios farmacéuticos (60.852), las aseguradoras de salud (2.178) y los hospitales (6.610). La significación es del 0,004.

Las aseguradoras de salud reciben estadísticamente una peor calificación en su página de Facebook. Sobre un máximo de 5 puntos, de media consiguen un 2,99, en comparación con el siguiente grupo con una nota más baja, los hospitales, que obtienen un 4,29, y la media del conjunto, que alcanza el 4,07. La significación es del 0,009. El conjunto con una mejor opinión son las empresas de tecnología, que obtienen un 4,80, seguidos por las de dispositivos biomédicos $(4,60)$, los fabricantes de productos sanitarios $(4,45)$ y los laboratorios farmacéuticos $(4,40)$.

Las empresas de tecnología también consiguen un mayor número de "me gusta" en sus publicaciones. De media obtienen 141,86, en comparación con la media general, que se sitúa en 54,36 . Después de estas compañías se hallan los fabricantes de productos sanitarios (media=90,50), los laboratorios farmacéuticos $(66,89)$, las empresas de dispositivos biomédicos $(35,50)$ y las aseguradoras de salud $(18,70)$. El conjunto con menos "me gusta" a las entradas son los hospitales, que arrojan de media 4,29. La relación es significativa al 0,000.

En cuanto a los comentarios a las publicaciones, las empresas de tecnología se sitúan en primer lugar, con una media de 3,00 . Después de hallan los fabricantes de productos sanitarios, con 2,81, y los hospitales, con 2,68. Los laboratorios farmacéuticos $(1,81)$, las aseguradoras de salud $(1,00)$ y, sobre todo, las empresas de dispositivos biomédicos $(0,50)$ están muy por detrás de la media general $(2,19)$. La relación es estadística al 0,043.

A pesar de que los centros hospitalarios se encuentran a la cola en el número de "me gusta" al perfil y a las publicaciones, se hallan a la cabeza en las entradas compartidas. De media, esta agrupación obtiene 28,63 shares, aunque le siguen de cerca las empresas de tecnología, con 24,29. Los laboratorios farmacéuticos se sitúan en tercera posición, con 22,57, y los fabricantes de productos sanitarios, en cuarta, con 16,59. Las aseguradoras de salud $(9,68)$ y las empresas de dispositivos biomédicos $(5,00)$ obtienen los peores resultados. La relación es significativa al 0,015. La media de todos ellos es 20,20.

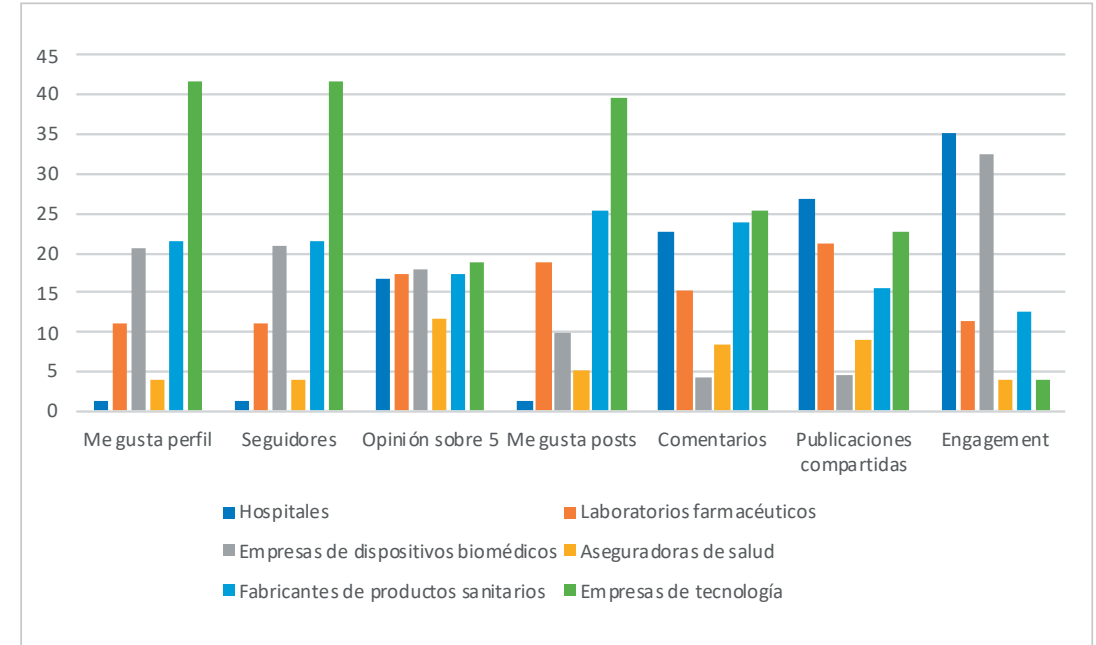

Gráfico 3. Actividad en Facebook.

Lo mismo sucede con el compromiso o engagement. Los hospitales se encuentran a la cabeza, con una media de 1,75. Por detrás están las empresas de dispositivos biomédicos $(1,62)$. Con bastante diferencia se hallan el resto: fabricantes de productos sanitarios $(0,63)$, laboratorios farmacéuticos $(0,57)$, aseguradoras de salud $(0,20)$ y las empresas de tecnología $(0,12)$. La media de todos ellos es 0,80. La relación es significativa al 0,000.

\subsection{Actividad en Twitter}

En Twitter no se han detectado diferencias estadísticamente significativas entre el número de publicaciones comparando los diferentes conjuntos de entidades. Las empresas de tecnología incluyen una media de 9.243 entradas, por delante de las aseguradoras de salud (5.752), fabricantes de productos sanitarios (5.651), hospitales (4.611), laboratorios farmacéuticos (4.328) y empresas de dispositivos biomédicos (2.197). El promedio general se sitúa en 5.100.

Sí se han hallado diferencias significativas entre el tipo de entidad y el número de perfiles a los que se sigue. La cantidad más alta es de las empresas de tecnología con 2.213, seguidas por las de dispositivos biomédicos (1.647) y las aseguradoras de salud (1.076). El resto está por debajo del millar de cuentas seguidas: los fabricantes de productos sanitarios siguen a una media de 941 , los laboratorios farmacéuticos a 827 , y los hospitales a 785 . La relación es significativa al 0,011. La media general es 962 . 
Algo similar sucede con el número de seguidores. Las empresas de tecnologías son las que más adeptos tienen, concretamente 55.206 de media. Es una cifra muy alejada del promedio general, que baja hasta los 10.377. Después están las aseguradoras de salud con 10.147, los fabricantes de productos sanitarios con 9.197, y los laboratorios farmacéuticos con 8.053. En la cola están los hospitales (4.324) y las empresas de dispositivos biomédicos (3.195). La significación es del 0,000 .

Al igual que sucedía con Facebook, sorprende que los hospitales pasen de la cola a la cabeza en algunas variables. Una de ellas es la media de "me gusta" a las publicaciones en Twitter; alcanzan un promedio de 10,77, cuando el general se queda en 7,68. Por detrás están los laboratorios farmacéuticos $(7,79)$, las empresas de tecnología $(7,13)$, los fabricantes de productos sanitarios $(6,36)$ y las aseguradoras de salud $(4,28)$. En último lugar se hallan las empresas de dispositivos biomédicos (2,00). La relación es significativa al 0,003.

Los hospitales también están en primer lugar en el número de retweets. Frente a la media global de 3,40 por publicación, estos centros logran 5,23. Muy por debajo de esa cifra se encuentran los laboratorios farmacéuticos $(3,24)$ y las empresas de tecnología $(3,00)$. Los que consiguen menos posts compartidos son los fabricantes de productos sanitarios $(2,79)$, las aseguradoras de salud $(1,59)$ y, sobre todo, las empresas de dispositivos biomédicos $(0,50)$. La relación es significativa al 0,000.

La media en conjunto de compromiso o engagement se sitúa en 0,310. Nuevamente los hospitales encabezan esta variable, con un 0,684 . Muy por detrás están los laboratorios farmacéuticos $(0,223)$, los fabricantes de productos sanitarios $(0,148)$, las aseguradoras de salud $(0,129)$, las empresas de tecnología $(0,115)$ y las de dispositivos biomédicos $(0,080)$. La significación es del 0,000.

Las empresas de tecnología incluyen más listas que los otros grupos. De media comparten 5,50, muy por encima del 1,75 del promedio general. Por detrás están las aseguradoras de salud $(2,50)$, hospitales $(1,72)$, fabricantes de productos sanitarios $(1,36)$, laboratorios farmacéuticos $(1,11)$ y empresas de dispositivos biomédicos $(0,00)$. La significación es del 0,019.

Las empresas de tecnología también proporcionan más fotos y vídeos a sus seguidores. Frente a los 1.382 documentos de este tipo que arrojan todas las entidades en conjunto, este tipo de compañías ofrecen de promedio 2.770. Después se encuentran las

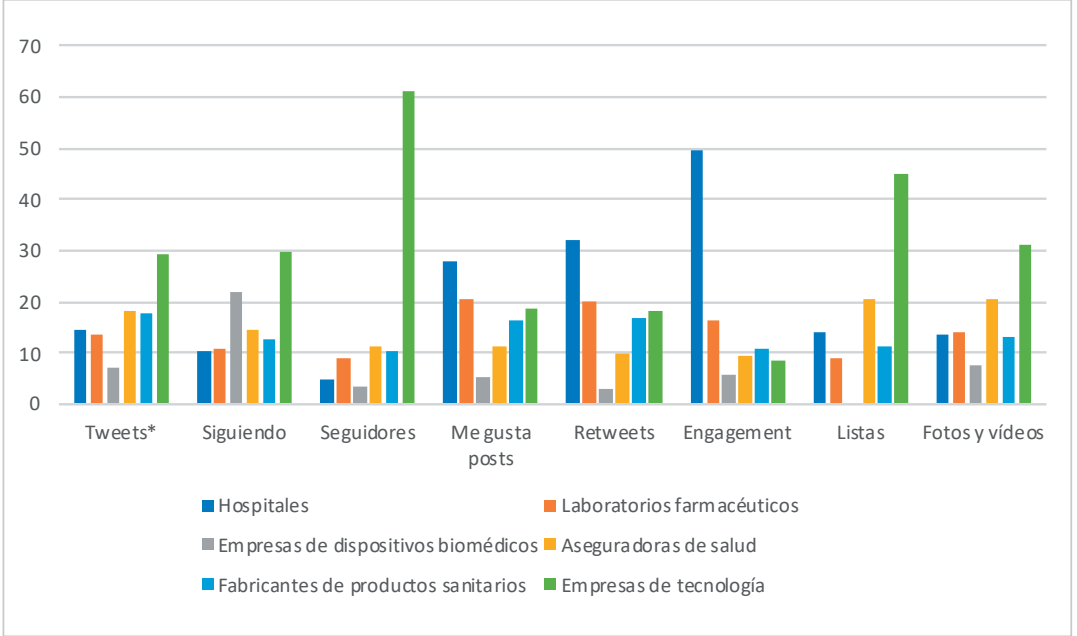

Gráfico 4. Actividad en Twitter.

*No se han encontrado diferencias estadísticamente significativas.

aseguradoras de salud (1.828), laboratorios farmacéuticos (1.252), y hospitales (1.211). En última posición están las empresas de dispositivos biomédicos, con una media de 691 fotos y vídeos. La significación es del 0,012.

\subsection{Actividad en YouTube}

Las empresas de tecnología publican más vídeos en YouTube que el resto. Frente a la media global de 162, estas entidades incluyen de media 723. Muy por detrás están los hospitales con un promedio de 201, los fabricantes de productos sanitarios con 166, y las empresas de dispositivos biomédicos con 132. Los que menos vídeos incluyen en esta red social son las aseguradoras de salud (98) y sobre todo los laboratorios farmacéuticos (70). La significación es del 0,021.

Quizá debido a un número más alto de vídeos publicados, las empresas de tecnología consiguen una suma mayor de visualizaciones totales. De media obtienen 4.840.771. Los siguientes son las aseguradoras de salud (3.237.792), y los fabricantes de productos sanitarios (1.847.720). Por debajo del promedio general (1.689.188) están las empresas de dispositivos biomédicos (1.639.328), laboratorios farmacéuticos (706.744) y hospitales (424.315). La significación es del 0,012 .

Aparte de colgar más vídeos, las empresas de tecnología tienen más seguidores que el resto. Arrojan un promedio de 8.873, cuando la media general es del 2.924. Con casi menos de la mitad, concretamente 4.502 , se hallan los fabricantes de productos sanitarios. Las empresas de dispositivos biomédicos, aunque con menos seguidores, se sitúan por encima de la media general, en concreto con 3.176. El resto se sitúan muy por debajo: 1.589 adeptos de media para los hospitales, 1.542 para los laboratorios farmacéuticos y 1.498 para las aseguradoras de salud. La significación es del 0,008.

En cambio, si se analiza la media de visualizaciones a cada vídeo, las empresas de tecnología bajan hasta el segundo lugar, con un promedio de 2.533. El grupo que en este caso consigue una mejor cifra son las aseguradoras de salud (5.242), a pesar de que son las que menos seguidores poseen y las segundas con menos vídeos publicados. El resto se 
halla por debajo de la media general (1.975): fabricantes de productos sanitarios (1.795), hospitales (307) y empresas de dispositivos biomédicos (278). La significación es del 0,021.

Dado que las aseguradoras de salud son las que más visualizaciones consiguen pese a ser las segundas con un menor número de vídeos divulgados, la tasa media de visualizaciones se encuentra muy por delante de la media general y del resto de grupos. En concreto este conjunto arroja una tasa de 1.871 visualizaciones, en comparación con el promedio global, que baja hasta los 393. Después están los laboratorios farmacéuticos con 155, los fabricantes de productos sanitarios con 117, y las empresas de tecnología con 112. Muy por debajo se sitúan los hospitales (89) y las empresas de dispositivos biomédicos (6,9). La significación es del 0,011.

Los hospitales, que no sobresalen por publicar muchos vídeos ni conseguir un alto número de seguidores o visualizaciones, son los que más "me gusta" consiguen en cada vídeo. De media obtienen 19, cuando el promedio global es de 6,63. Las empresas de tecnología se encuentran en segundo lugar con un 8,13 , y los fabricantes de productos sanitarios en tercera posición con 5,13. El resto está muy por debajo de la primera cifra: 4,76 para las aseguradoras de salud, 4,16 los laboratorios farmacéuticos y 3,00 las empresas de dispositivos biomédicos. La significación es del 0,001.

Además de contar con más "me gus-

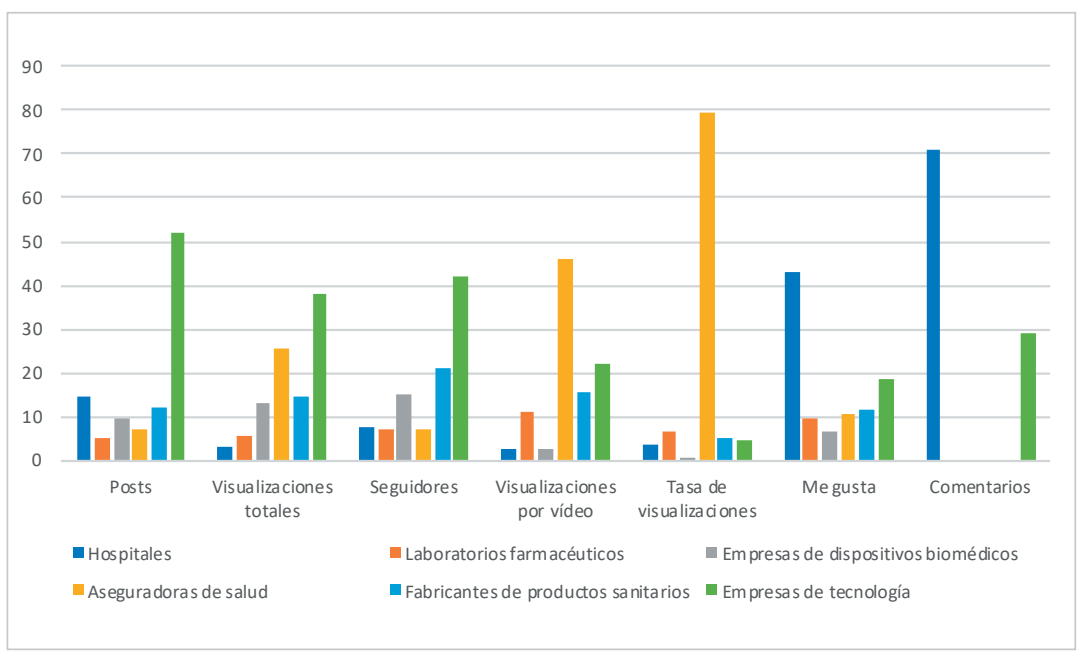

Gráfico 5. Actividad en YouTube.

ta", los hospitales también consiguen más comentarios a sus vídeos. De media obtienen 0,36, en comparación con el siguiente conjunto con más mensajes: las empresas de tecnología, que alcanzan 0,15. En el resto de los casos la media es de 0,00. La significación es del 0,000.

\subsection{Actividad en Instagram}

En Instagram se han analizado las medias de las publicaciones, los seguidores y los perfiles seguidos. Únicamente en la segunda variable se han detectado diferencias significativas. Las empresas de tecnología son las que consiguen un mayor número de adeptos: de media, 31.198. El siguiente grupo con más seguidores son los fabricantes de productos sanitarios, que poseen menos de un tercio, 10.057. Los laboratorios farmacéuticos (8.221), las aseguradoras de salud (3.084) y los hospitales (2.899) tienen considerablemente menos. La media general es 31.198 y la significación, 0,003. Hay que señalar que ninguna empresa de dispositivos biomédicos cuenta con un perfil en Instagram.

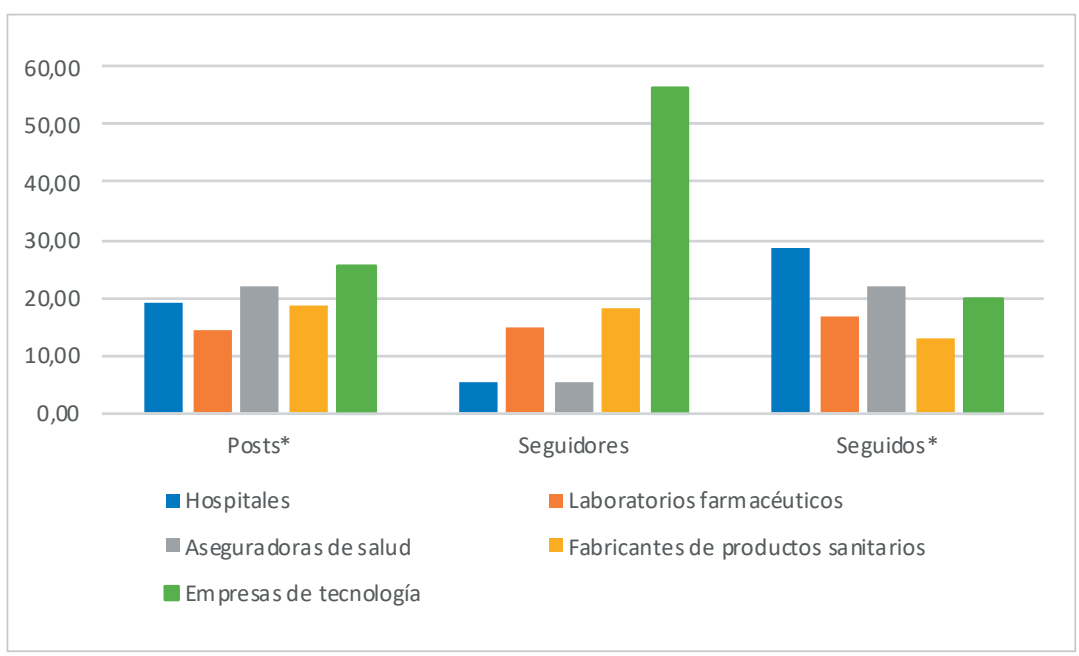

Gráfico 6. Actividad en Instagram.

* No se han encontrado diferencias estadísticamente significativas.

\section{Conclusiones}

En este trabajo se han analizado los perfiles en Facebook, Twitter, YouTube e Instagram de las 240 entidades relacionadas con la salud que aparecen divididas en 6 grupos en el Monitor de reputación sanitaria de 2017: hospitales, laboratorios farmacéuticos, fabricantes de productos sanitarios, aseguradoras de salud, empresas de tecnología y empresas de dispositivos biomédicos más reputadas que operan en España.

Se ha comprobado que las aseguradoras de salud están más presentes en las redes sociales que el resto (con una tasa media de 3,12 redes sociales por entidad), seguidas por los fabricantes de productos sanitarios $(2,75)$ y las empresas de tecnología $(2,70)$. A la cola se encuentran los laboratorios farmacéuticos $(2,58)$, las empresas de productos biomédicos 
$(1,80)$ y, sobre todo, los hospitales $(0,92)$. El primer conjunto de entidades es en general más activo dentro de estas plataformas que el segundo; es decir, incluyen más publicaciones, más fotos y vídeos, etc.

Las empresas de tecnología son las que tienen una mayor popularidad en las redes sociales, pero únicamente a nivel superficial. Son las que poseen más seguidores y las que reciben un mayor número de "me gusta" a los perfiles, pero cuando se profundiza en la actividad de los internautas, se observa que en general los hospitales obtienen un mayor engagement o compromiso. El público incluye considerablemente más comentarios y más "me gusta" a las entradas que publican estos centros. Asimismo comparten con más frecuencia sus divulgaciones.

Se observa por tanto que los hospitales cuentan con una mayor interacción por parte de la audiencia, a pesar de que son menos asiduos y menos activos en las redes sociales. De esta manera se puede determinar que una mayor presencia y actividad en las redes sociales en el ámbito sanitario puede influir en el número de seguidores, pero no se correlaciona con una mayor interacción por parte del público.

Dado el interés que el público tiene hacia la información que los hospitales divulgan en redes sociales, se recomienda a estos centros aumentar su presencia y actividad en estas plataformas, ofreciendo información no sólo corporativa, sino también de interés general, con contenidos fiables y de calidad.

\section{Limitaciones}

Esta investigación se ha centrado en el paradigma cuantitativo, y conviene señalar que los tamaños de los 6 tipos de entidades sanitarias analizadas son muy diferentes, siendo 100 el mayor (hospitales) y 5 el menor (dispositivos biomédicos), por lo que los resultados y sus conclusiones deben tratarse con precaución. Para tener una mayor comprensión de las fuentes y del tipo de información sobre salud y sanidad que circulan en las redes sociales, se recomienda continuar con la misma línea de análisis, ampliando la muestra, incluyendo nuevos parámetros de estudio y acometiendo análisis cualitativos. Así también se podrá profundizar en los intereses y el consumo por parte del público de la información relacionada con la salud en internet.

\section{Los hospitales cuentan con una mayor interacción por parte de la audiencia, a pesar de que son menos asiduos y me- nos activos en las redes sociales}

\section{Referencias}

Alkhateeb, Fadi M.; Clauson, Kevin A.; Latif, David A. (2011). "Pharmacist use of social media”. International journal of pharmacy practice, v. 19, n. 2, pp. 140-142.

https://doi.org/10.1111/j.2042-7174.2010.00087.x

Análisis e investigación (2017). Monitor de reputación sanitaria (MRS).

http://www.merco.info/es/monitor-reputacion-sanitaria-hospitales

Benetoli, Arcelio; Chen, Timothy F.; Schaefer, Marion; Chaar, Betty; Aslani, Parisa (2017). “Do pharmacists use social media for patient care?". International journal of clinical pharmacy, v. 39, n. 2, pp. 364-372.

https://doi.org/10.1007/s11096-017-0444-4

Benítez-Berrocal, Marta; Faba-Pérez, Cristina (2016). "La presencia de las redes sociales en el entorno sanitario: el caso de los hospitales mejor posicionados del mundo". Ibersid: revista de sistemas de información y documentación, v. 10, n. 2, pp. 21-30.

https://ibersid.eu/ojs/index.php/ibersid/article/view/4334/3849

Costa-Sánchez, Carmen; Míguez-González, María-Isabel (2018). “Use of social media for health education and corporate communication of hospitals". El profesional de la información, v. 27, n. 5, pp. 1.145-1.154.

https://doi.org/10.3145/epi.2018.sep.18

Costa-Sánchez, Carmen; Túñez-López, Miguel; Videla-Rodríguez, José-Juan (2016). “Hospitales españoles en la web social. Gestión de Facebook y Twitter por el Hospital Sant Joan de Déu (Barcelona)". Revista latina de comunicación social, v. 71, pp. 1.108-1.130.

https://doi.org/10.4185/RLCS-2016-1137

Crilly, Philip; Hassanali, Wasim; Khanna, Gary; Matharu, Kiranjit; Patel, Deep; Patel, Disha; Rahman, Fahmida; Kayyali, Reem (2018). "Community pharmacist perceptions of their role and the use of social media and mobile health applications as tools in public health". Research in social and administrative pharmacy, v. 15, n. 1. 
https://doi.org/10.1016/j.sapharm.2018.02.005

España (2003). “Ley 16/2003, de 28 de mayo, de cohesión y calidad del Sistema Nacional de Salud”. BOE, n. 128, 29 de mayo, pp. 20.567-20.588.

http://www.boe.es/boe/dias/2003/05/29/pdfs/A20567-20588.pdf

España (2009). "Real decreto 1591/2009, de 16 de octubre, por el que se regulan los productos sanitarios”. BOE, n. 268, 6 de noviembre.

https://www.boe.es/buscar/pdf/2009/BOE-A-2009-17606-consolidado.pdf

España (2010). “Real decreto 824/2010, de 25 de junio, por el que se regulan los laboratorios farmacéuticos, los fabricantes de principios activos de uso farmacéutico y el comercio exterior de medicamentos y medicamentos en investigación". BOE, n. 165, 8 de julio.

https://www.boe.es/buscar/act.php?id=BOE-A-2010-10827

Fenin (s.f.). Quiénes somos. Federación Española de Empresas de Tecnología Sanitaria.

https://www.fenin.es/about_us

Fox, Susannah (2014). "The social life of health information". Pew Research Center, Jan. $15^{\text {th }}$. http://www.pewresearch.org/fact-tank/2014/01/15/the-social-life-of-health-information

Glover, McKinley; Khalilzadeh, Omid; Choy, Garry; Prabhakar, Anand M.; Pandharipande, Pari V.; Gazelle, G. Scott (2015). "Hospital evaluations by social media: A comparative analysis of Facebook ratings among performance outliers". Journal of general internal medicine, v. 30, n. 10, pp. 1440-1446.

https://doi.org/10.1007/s11606-015-3236-3

Griffis, Heather M.; Kilaru, Austin S.; Werner, Rachel M.; Asch, David A.; Hershey, John C.; Hill, Shawndra; Ha, Yoonhee P.; Sellers, Allison; Mahoney, Kevin; Merchant, Raina M. (2014). “Use of social media across US hospitals: Descriptive analysis of adoption and utilization". Journal of medical internet research, v. 16, n. 11, p. 264.

https://doi.org/10.2196/jmir.3758

Health Research Institute (2012). Social media "likes" healthcare. From marketing to social business.

https://www.pwc.com/us/en/health-industries/health-research-institute/publications/pdf/health-care-social-media-report.pdf

Health Union (2016). Social media for health: What socially-active patients really want.

https://health-union.com/wp-content/uploads/2016/06/Social-Media-for-Health.pdf

IAB Spain (2017). Estudio anual de redes sociales. IAB Spain.

https://iabspain.es/wp-content/uploads/iab_estudioredessociales_2017_vreducida.pdf

İlgün, Gülnur; Uğurluoğlu, Özgür (2018). "How Turkish private hospitals use social media: A qualitative study". Journal of social service research, v. 45, n. 1.

https://doi.org/10.1080/01488376.2018.1479339

Martínez-Millana, Antonio; Fernández-Llatas, Carlos; Basagoiti-Bilbao, Ignacio; Traver-Salcedo, Manuel; Traver-Salcedo, Vicente (2017). "Evaluating the social media performance of hospitals in Spain: A longitudinal and comparative study". Journal of medical internet research, v. 19, n. 5, e181.

https://doi.org/10.2196/jmir.6763

Medina-Aguerrebere, Pablo (2018). "El rol de las redes sociales en la comunicación de marca de los hospitales españoles". adComunica, v. 15, pp. 215-233.

https://doi.org/10.6035/2174-0992.2018.15.11

Medina-Aguerrebere, Pablo; Buil-Gazol, Pilar; Heath, Robert L. (2015). "Brand dissemination in Canadian hospitals through Facebook". The international journal of communication and health, v, 7, pp. 27-39.

http://communicationandhealth.ro/upload/number7/PABLO-MEDINA.pdf

Ministerio de Sanidad Consumo y Bienestar Social (2018). Catálogo nacional de hospitales. Gobierno de España. https://www.mscbs.gob.es/ciudadanos/prestaciones/centrosServiciosSNS/hospitales/docs/2018_CNH.pdf

Smith, Katherine-Taken (2017). “Hospital marketing and communications via social media”. Services marketing quarterly, v. 38, n. 3, pp. 187-201.

https://doi.org/10.1080/15332969.2017.1363518

Timian, Alex; Rupcic, Sonia; Kachnowski, Stan; Luisi, Paloma (2013). “Do patients 'like' good care? Measuring hospital quality via Facebook". American journal of medical quality, v. 28, n. 5, pp. 374-382.

https://doi.org/10.1177/1062860612474839 
Tyrawski, Jennifer; DeAndrea, David C. (2015). “Pharmaceutical companies and their drugs on social media: A content analysis of drug information on popular social media sites". Journal of medical internet research, v. 17, n. 6, e130. https://doi.org/10.2196/jmir.4357

Unespa (2017). Informe estamos seguros. Asociación Empresarial del Seguro.

https://unespa-web.s3.amazonaws.com/main-files/uploads/2018/06/Informe-2017-Estamos-Seguros_V05.pdf

Unión Europea (2017). “Reglamento (UE) 2017/745 del Parlamento Europeo y del Consejo, de 5 de abril de 2017, sobre los productos sanitarios, por el que se modifican la Directiva 2001/83/CE, el Reglamento (CE) n. 178/2002 y el Reglamento (CE) n. 1223/2009 y por el que se derogan las Directivas 90/385/CEE y 93/42/CEE del Consejo". Diario oficial de la Unión Europea, n. 117, de 5 de mayo, pp. 1-175.

https://eur-lex.europa.eu/legal-content/ES/TXT/?uri=celex\%3A32017R0745

WEGO Health (2017). Role of patient influencers: How do patients truly share information?. Wego Healt Solutions. https://www.wegohealth.com/wp-content/uploads/2017/05/PT-2-WEGO-Health-Solutions_BIS_sharing-behavior.pdf

Wong, Charlene A.; Ostapovich, Gabrielle; Kramer-Golinkoff, Emily; Griffis, Heather; Asch, David A.; Merchant, Raina M. (2016). "How U.S. children's hospitals use social media: A mixed methods study". Healthcare, v. 4, n. 1, pp. 15-21. https://doi.org/10.1016/j.hjdsi.2015.12.004

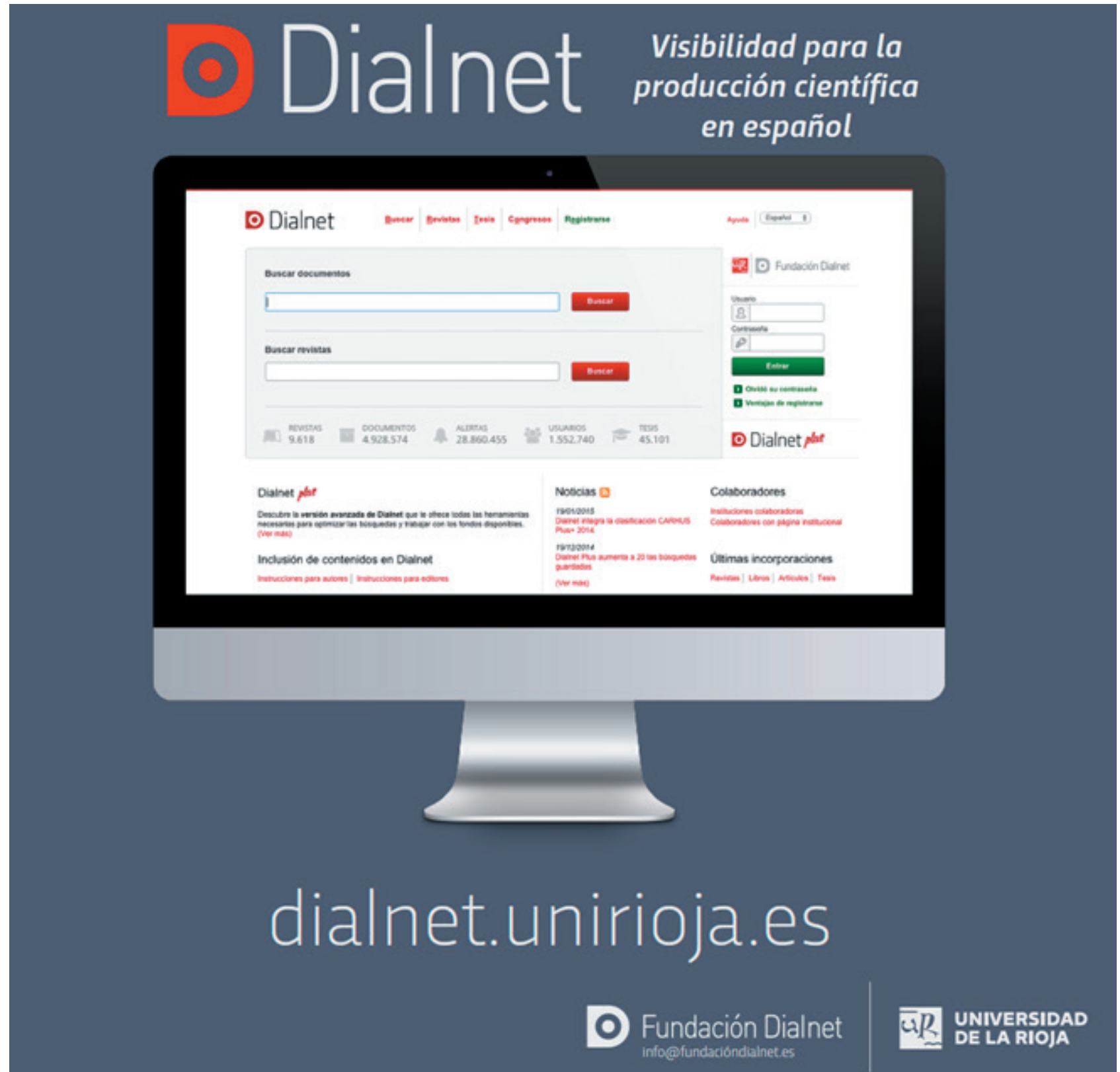

